Document downloaded from:

http://hdl.handle.net/10251/65378

This paper must be cited as:

Báguena Albaladejo, M.; Tavares De Araujo Cesariny Calafate, CM.; Manzoni ., P.; Cano Escribá, JC.; Manzoni, P. (2013). Assessing the Impact of Obstacle Modeling Accuracy on IEEE 802.11p Based Message Dissemination. Third ACM International Symposium on Design and Analysis of Intelligent Vehicular Networks and Applications (DIVANet 13). ACM. doi:10.1145/2512921.2512929.

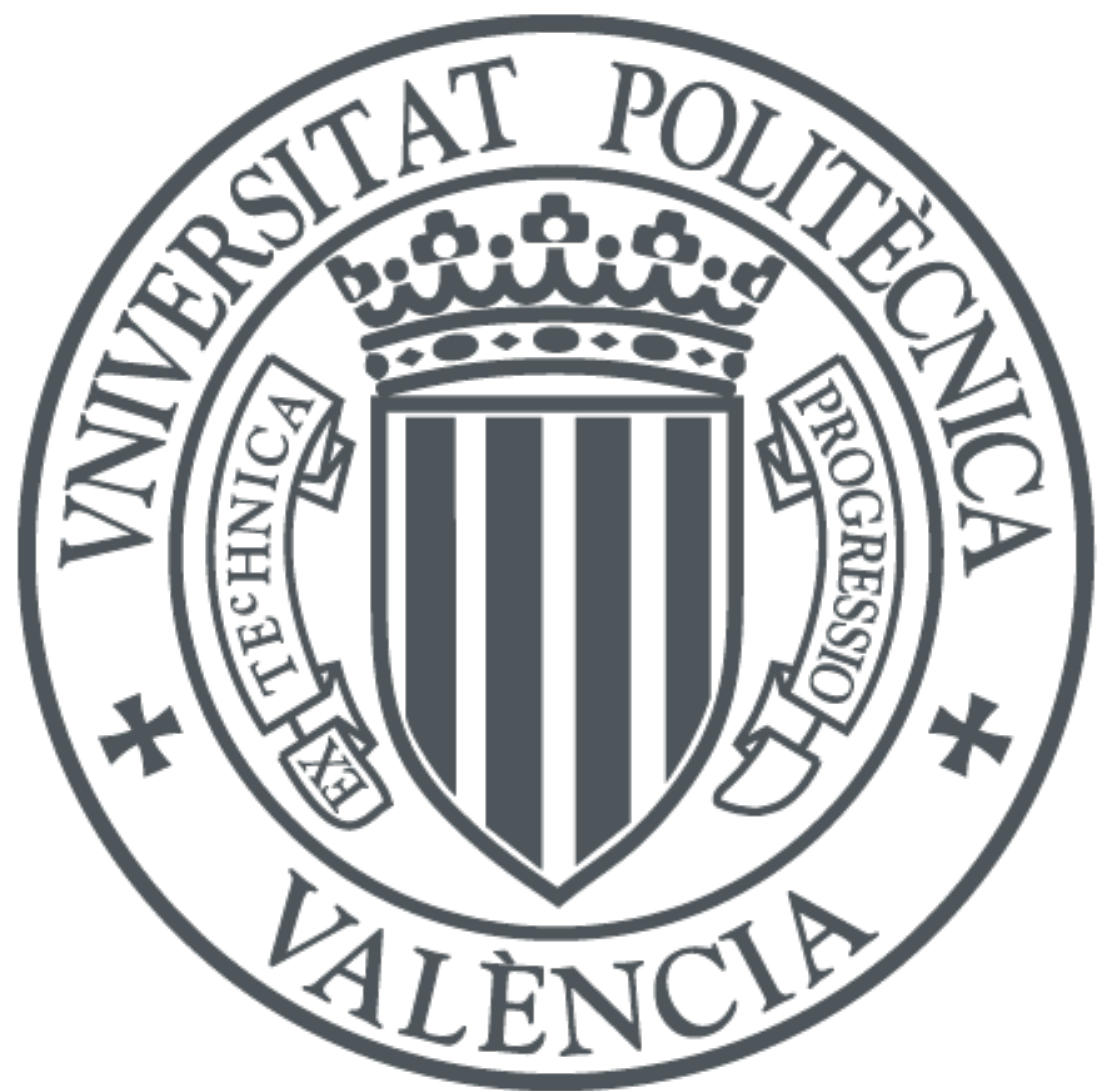

The final publication is available at

Copyright ACM

Additional Information

(C) ACM 2013. This is the author's version of the work. It is posted here for your personal use. Not for redistribution. The definitive Version of Record was published in ACM, In Proceedings of the third ACM international symposium on Design and analysis of intelligent vehicular networks and applications (pp. 123-128).

http://dx.doi.org/10.1145/2512921.2512929 


\title{
Assessing the impact of obstacle modeling accuracy on IEEE 802.11p based message dissemination
}

\author{
Miguel Báguena, Carlos T. Calafate, Juan-Carlos Cano, Pietro Manzoni \\ Department of Computer Engineering \\ Universitat Politècnica de València \\ Camino de Vera S/N, 46022, Spain. \\ mibaal@upvnet.upv.es, \{calafate, jucano, pmanzoni $\} @$ disca.upv.es
}

February 21, 2014

\begin{abstract}
Deploying real IEEE $802.11 \mathrm{p}$ vehicular network testbeds is a challenging but difficult option for most researchers. In these cases, the research community relies on simulation tools to test their protocols. However, since simulation accuracy is a critical issue, real testbed results should be used as a reference to improve simulation behavior. Our proposal adjusts common propagation models to mimic samples taken from real environments, and it uses a building aware model to achieve as much accuracy as possible in urban scenarios. We evaluate the performance differences obtained with this model against other usual simulation schemes, like line-of-sight propagation models, models where no building blockage is taken into account, and models where propagation is only allowed along streets, achieving differences of up to $70 \%$ in some measurements. Going a step further, the model is used to study the radio propagation behavior along different city layouts, showing that the actual building layout is one of the key factors affecting protocol performance in urban environments.
\end{abstract}

\section{Introduction}

New devices compliant with the 802.11 p standard are being developed by several hardware companies [1]. This enables researchers to start obtaining real per- formance results [2] and make them available for the research community [3]. There are also some current [4] and future [5] projects attempting to develop real testbeds to allow deploying software for performance testing. However, these initiatives are either too expensive, too closed or too ethereal to serve the purposes of many researchers. Therefore, they must rely on simulations to check the efficiency of their protocols.

One of the key pieces of a simulator is the propagation model, especially when addressing vehicular networks. There are some works that focus on this problem, such as Killat et al. [6], whose model adds vehicle traffic density to the Nakagami model; CORNER [7], which uses road topology to work out the received power accordingly; Hosseini Tabatabaei et al. [8], whose model enhances the two-ray-ground model to take into account reflections from buildings; Boban et al. [2], whose model is aware of non line-of-sight conditions to calculate the final power of a signal; Martinez et al. [9], whose hybrid model allows lineof-sight transmissions for two vehicles if they are in the same or adjacent streets, blocking signals otherwise; and Sommer et al. [10], whose hybrid model relies on obstacle information to heavily penalize any transmission traversing walls. The work of Cozzetti et. al. [11], who present RUG to improve current corner-based propagation models, and Scopigno et. al. [12], whose RADII uses a ray tracing technique 
to simulate the propagation for any building layout, are also relevant in this context. However, most of them are not aware of the building topology, they are not based on real measurements, or they are only focused on certain topologies or situations.

Once a simulator is realistic enough, we can close the circle. We can assess the effectiveness of any proposal in many different cities by first studying how a simpler solution performs in a specific scenario. Going one step further, we can study different simulations to determine the key factors affecting message propagation in a city, such as road shapes, obstacles, road interconnections, etc. This offers researchers, designers and developers the most immediate knowledge of how their protocol will perform under certain conditions, allowing them to tune or modify it properly, or to work out a new solution.

In this paper we tune common propagation models to produce results that resemble the real testbed measurements in [3]. This tuned model is combined with the buildings aware model presented in [10] to provide a model able to offer a realistic outcome, regardless of line-of-sight or non-line-of-sight conditions, realistic or synthetic road topologies, or highway or urban environments. After assessing its properties, the model is used to evaluate different city layouts in order to find the key factors that have significant effects in the final behavior of a protocol.

The paper is organized as follows: in section 2 the proposed model is compared against simpler existing models to highlight the performance differences detected when simulating vehicular scenarios. In section 3 different cities are evaluated, and the factors affecting performance are identified. Finally, section 4 concludes the paper.

\section{Model tuning and perfor- mance assessment}

In this section we extend the model presented in [13], adjusting the signal propagation model parameters according to real-life experiments. This propagation model is made of two different components. The first one deals with signal propagation in free space with- out or with small mobile obstacles, like cars. It is based on a Free Space model and a Nakagami model whose $\alpha$ (Free Space's attenuation coefficient) and $m$ (Nakagami's shape factor) propagation parameters can be tuned in order to get a behavior as close to reality as possible. The second component takes advantage of the model by Sommer et. al. [10] to show a realistic behavior in the presence of buildings. Combining these two models, not only regular signal propagation situations are properly simulated, but also the presence of buildings is simulated accurately.

We take special care about $\alpha$ and $m$ propagation parameters. Using this model, we will assess the impact of buildings and other obstacles on communications. These studies were performed using the same methodology described in our previous paper.

\subsection{Channel model tuning}

In this section we aim at replicating real life transmission conditions in $802.11 \mathrm{p}$ vehicular environments. We aim at tuning two different models. The first one is the Vehicles as obstacles model, which simulates common urban vehicular communication where mobile obstacles partly block the radio signal. The second one is the Line of sight model, which simulates the simplest transmission situation, where two vehicles can communicate when in line-of-sight.

As a reference for the first model we take the experimental results provided by Meireles et al. [3] for vehicle obstructed conditions. We establish the reference for the second model in order to get a relative reference for data concerning vehicles as obstacles. This reference is based on the line-of-sight model in [3], and the configuration presented in [14]. Notice that data presented in both works is compatible and complementary.

Our proposal combines the Modified Free Space model and the Nakagami model. In a first step we tune our model by adjusting the $\alpha$ and $m$ parameters. In the simulated scenario used to adjust our model, two nodes are deployed respecting line-of-sight restrictions. We took different measurements of the received packet ratio by varying the distance between the nodes from one case to another. 


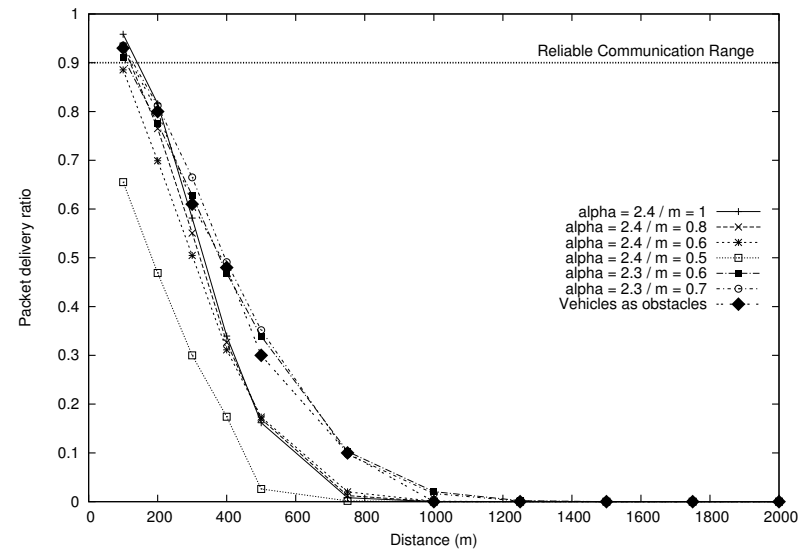

Figure 1: Similarity of Vehicles as obstacles model and various $\alpha$ and $\mathrm{m}$ configurations

Since both $\alpha$ and $m$ parameters affect the received packet ratio, we simultaneously vary both values to get the best curve fitting. It was done for two both Line of sight and Vehicles as obstacles models.

Figure 1 shows the differences between several propagation models according to the value of the $\alpha$ and $m$ parameters and the Vehicles as obstacles results from [3]. We first focus on the $m$ values since they affect both the slope and the packet delivery ratio. The optimal curve slope corresponds to a value of 0.7 for the $m$ parameter because smaller values cause the slope to be too low, as can be seen in the figure. Focusing on the value of $\alpha$, we find that the value offering good accuracy in terms of packet delivery ratio is 2.3. As can be seen, there is a minimal error between the model and data in [3]: the squared error is lower than 0.006. We find that the reliable communication range ( $>90 \%$ of packet delivery ratio) of $100 \mathrm{~m}$ obtained in [3] is also maintained.

Figure 2 shows the differences between several propagation models according to the value of both $\alpha$ and $m$ parameters based on the line-of-sight results. This model will be used as reference in following the subsections that follow. If we focus on slope, values in the range from 1 to 1.1 offer a similar fading behavior. For the $\alpha$ parameter, the value of 2.2 is the best possible fit. Finally, we can see that the reliable

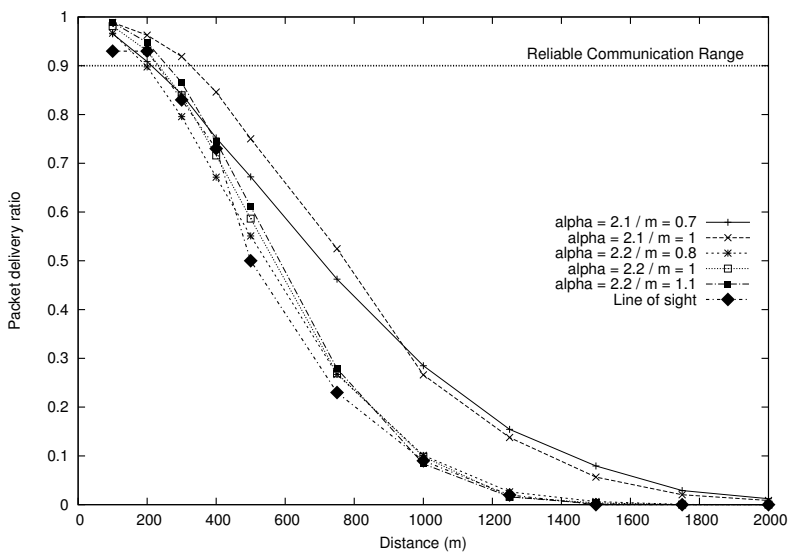

Figure 2: Similarity of Line of sight model and various $\alpha$ and $\mathrm{m}$ configurations

communication range in [3] is maintained, and that this model is also consistent with the reference value for the maximum transmission range defined in [14], with a packet delivery ratio lower than $5 \%$ at $1400 \mathrm{~m}$.

\subsection{Assessing the performance impact of static and mobile obstacles}

After adjusting the propagation model parameters in the previous section, and considering either line-ofsight or vehicle obstructions for the signal propagation, we now present a new set of experiments to evaluate two main features: (i) the impact of mobile obstacles (vehicles), and (ii) the impact of static obstacles (buildings) on message delivery. To evaluate these features we used $\mathrm{OMNeT}++$ combined with Sumo to simulate a scenario where 500 cars move following random routes within a $12 \mathrm{~km}^{2}$ area in the peripheral area of Moscow.

The propagation models have been tested in three different scenarios with different building layouts. The first one is the No buildings scenario, where the inexistence of buildings is assumed. The second one is the Real buildings scenario, where the real building layout, as defined in the OpenStreetMap database, is used. The third one is the Synthetic buildings scenario, where the building layout is such that radio 


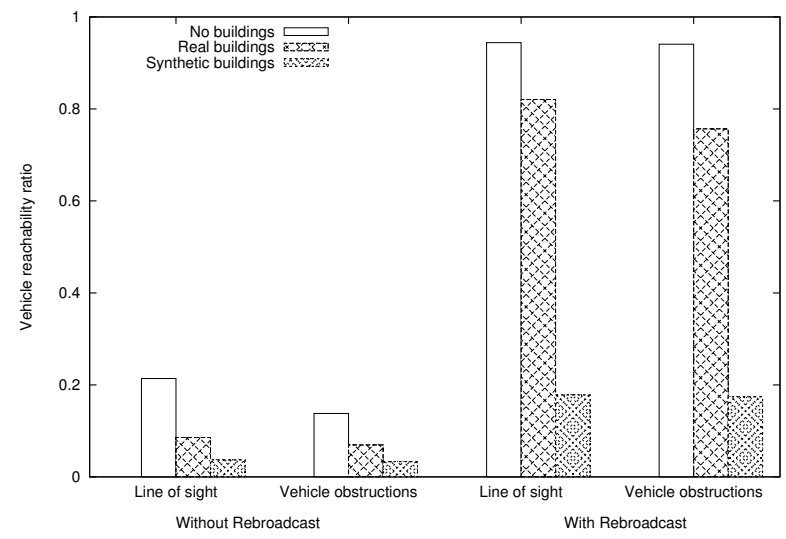

Figure 3: Impact of static obstacles under different conditions

transmissions only become possible along the roads. This scenario was included because this is a common assumption in current research.

In terms of traffic generation, all cars broadcast packets at regular intervals of $10 \mathrm{~s}$, and we consider two cases: (i) broadcasts are limited to one hop; and (ii) broadcasts are rebroadcasted by other vehicles (flooding).

\subsubsection{Impact of obstacles}

Figure 3 shows the average percentage of cars that are reachable in a one-hop beaconing process, with different obstacle models, in all scenarios. We can find a reduction in the Vehicles as obstacles model near to one third over the Line-of-sight model in the worst case. These differences will be a key factor in one-hop beaconing protocols, not only due to the final amount of neighbors detected, but also due to the quality of these links and the possible forwarders in a routing protocol algorithm.

This figure also reveals that the final vehicle reachability ratio highly depends on the existing buildings. Since Moscow is a city with a low building density, a simulation in a highly restrictive configuration will provoke a higher error degree compared to a less restrictive one. We have the opposite problem in a high building density scenario. Simulations with weak re-

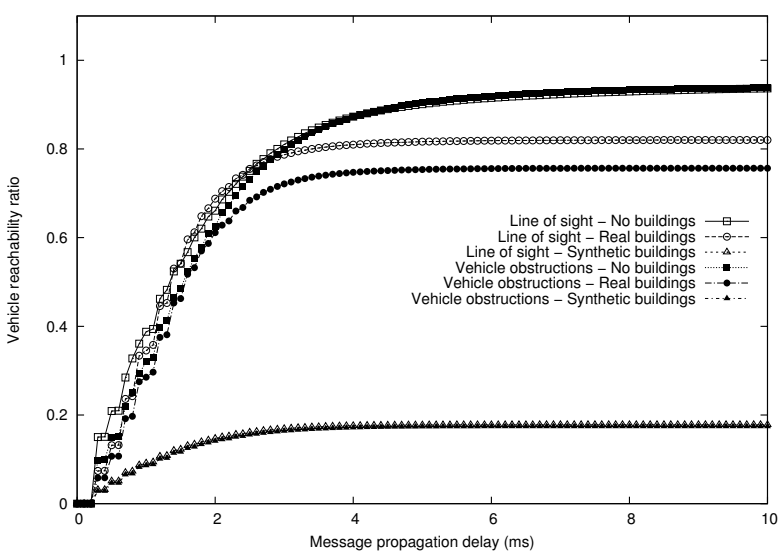

Figure 4: Vehicle reachability behavior along time

strictions also give us a high error degree. To avoid these problems, accurate building maps are needed.

Focusing on the broadcasting strategy, we can see that it evidences the effect of static obstacles because it amplifies its effects. With no rebroadcasting, only the nodes near the sender are reached (1 hop neighbors). However, in a multi-hop approach, the building obstruction effect is amplified at every hop, making it more evident. Results show that, except for the synthetic obstacles scenario, the flooding process is successful, reaching the majority of the vehicles circulating in the target area, as desirable.

\subsubsection{Delay study}

To analyze the flooding evolution in more detail, figure 4 shows the vehicle reachability behavior. We can observe here how the values presented in figure 3 are achieved along time, allowing to understand how the propagation model and the presence of static obstacles influences the perceived delay.

Observing the differences between the Line-of-sight model and the Vehicles as obstructions model, we see how a lower transmission radius causes the Vehicles as obstacles model to be always below the Line-ofsight model at any given time. The greatest difference occurs for the real building model, where buildings allow a moderate car reachability, as stated in previous subsections. Here we can find differences 
greater than half a millisecond for a car reachability of about $70 \%$.

However, the main impact in terms of performance is caused by the static obstacles factor. We can see clearly the delay increment that different static obstacles add when focusing on synthetic and real buildings. Not only a lower percentage of cars is reached, but also they are reached with a higher temporal cost. Therefore, we can conclude that an excessive or an insufficient building obstructiveness will lead our experiments to a variable error in terms of delay. In the following section we will examine other cities with different buildings densities to quantify how different values for this parameter can vary the final outcome shown by each of them. Since cities are a very complex environment, each having different road layouts and other parameters that can affect our results, in the sections that follow we will try to determine which are the main parameters affecting performance, isolating the effects of different building layouts.

\section{Evaluation of different city profiles}

As stated before, buildings block signal propagation causing transmission and connectivity failures. Since different cities present different building densities and layouts, every city will present a different connectivity degree. In this section we aim at evaluating this connectivity degree as a critical factor that could affect the final performance.

\subsection{Cities comparison}

We first selected a few cities to be used as a representative sample. The information for all the cities was extracted from the OpenStreetMap database. Despite correspondence to reality is not fully assured, the realism of these samples is much higher than what synthetic models can produce.

The test scenarios include residential areas, such as the Moscow maps presented before; grid areas, such as Washington DC; and urban canyon areas, such as Milan and Paris.

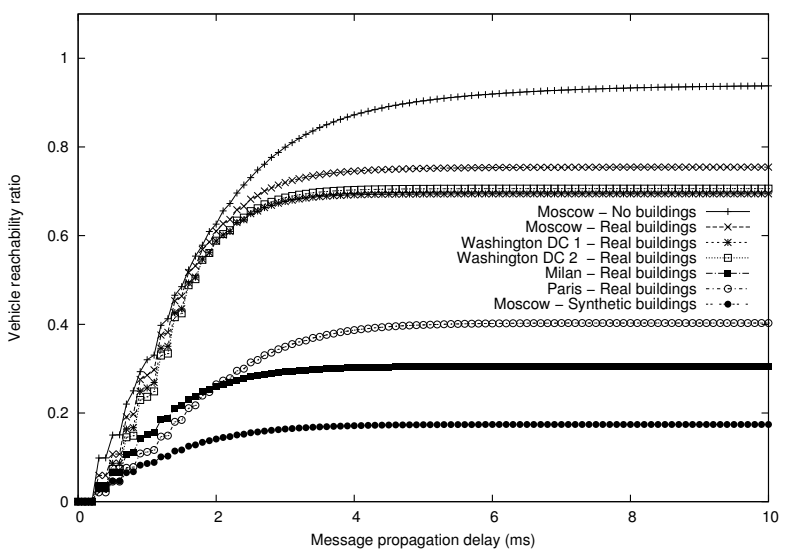

Figure 5: Vehicle reachability behavior along time for different city layouts

The conditions of the experiments are the same as above: we used OMNeT ++ combined with Sumo to simulate a scenario where 500 cars move by following random routes. In terms of traffic generation, all cars broadcast packets at regular intervals of $10 \mathrm{~s}$, and we consider the case where broadcasts are rebroadcasted by other vehicles (flooding). We tune our attenuation model with the parameters found according to the Vehicles as obstacles paradigm.

Figure 5 shows the vehicle reachability behavior along time for different city layouts. We also include there two reference series. The first of them is the Moscow version with no buildings. This curve shows a city where the impact of static obstacles is not taken into account. If all road layouts of all cities were the same, this would be the maximum value of reachability that a city may achieve. However, there are a lot of factors that also affect the final behavior, so it must only be used as a reference, and not as an upper bound. We also include a Moscow version using synthetic buildings in order to obtain a highly restrictive version, almost unreal, of the maximum obstacle blockage level. Similarly to the former one, this is used as a reference alone.

Looking at results we can see that different cities have different outcomes. In the figure, two groups are evident. The lower group is composed by cities 
which we intuitively associate to high density building areas, as can be our synthetic layout or the real downtown of European cities. However, the upper group, constituted by the Moscow and Washington cases, is more heterogeneous. The construction intensity varies significantly: from low density scenarios (Moscow scenario), and residential scenarios (first Washington scenario) to more dense scenarios (second Washington scenario).

This variable effect shown in different cases takes place because there are many factors that impact the final transmission effectiveness. Transmissions along roads are possible and relevant, in addition to transmissions to roads different from the source one. Therefore, not only obstacle density must be taken into account, but also the actual city road layout is important.

In the following subsection we will examine different factors to isolate the effect of building and road layout for our data set.

\subsection{Cities profiling and result analysis}

Fogue et al. [15], investigated some factors related to city street layouts, assessing their impact. They have identified a trend which relates message dissemination performance to the road layout and the vehicular density. Since in our analysis we introduced factors related to signal blockage by buildings, we aim at identifying a dependence in the performance of our sample of two main components: one of them will be related to the road layout, and another one will be related to the building layout. These two factors must define the trend in our experiments in order to effectively check the influence of obstacles in the final performance.

In order to isolate the effects that different building layouts have in the performance of our sample, we will examine all the cities that we have simulated in the previous subsection. We will examine the city maps as extracted from OpenStreetMaps to create a profile for each city, and identify a trend that ties the results of our simulations with these static measurements.

Table 1 shows some of the static measurements made over the OpenStreetMap definition files. Since obstacle blockage depends on building size, building

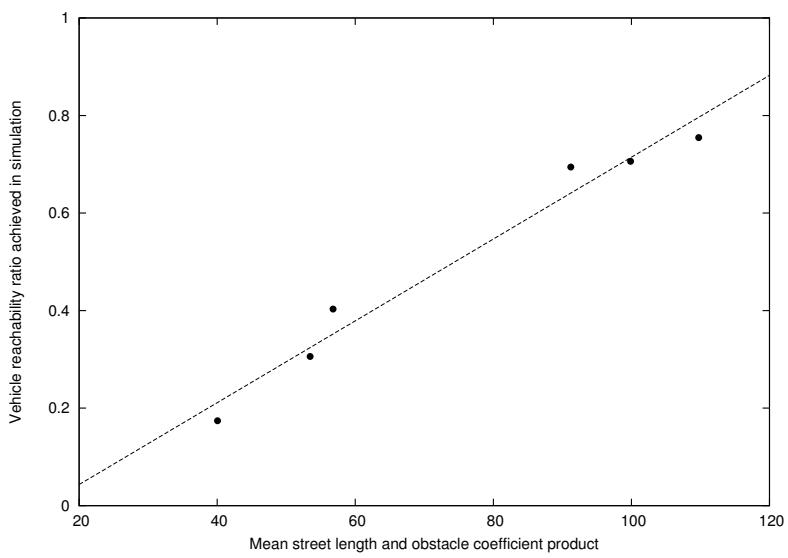

Figure 6: Combined street length and obstacle coefficient trend in our sample with lineal regression.

position, and other streets availability, we create a metric, called obstacle coefficient, that tries to group all these factors in a single a priori value. This value is the mean number of rays that, starting from a street, can reach a different one. We calculate it by defining random points in the street map, and checking whether streets different from the source one can be reached. With this coefficient we aim at providing a statistical approach of the building, as well as density and the blockage probability of a transmission to a vehicle in other streets.

To obtain a definitive metric, we propose selecting the average street length for the streets layout component, and the obstacle coefficient for the building layout component. The average street length is a good choice since it is directly proportional to the number of vehicles that are reachable along that street. In addition, a city density measure is also taken into account since smaller roads are usually related to a more dense and meandering city, and vice versa. This value is the ideal complement for our obstacle coefficient, only taking into account transmissions to different streets from the source one.

Figure 6 shows the trend that both the average street length and the obstacle coefficient form together. We can see that, for our sample, the street length and the obstacle coefficient are tied to the fi- 
Table 1: City profiles

\begin{tabular}{|c|c|c|c|c|c|c|c|}
\hline & $\begin{array}{c}\text { Moscow } \\
\text { No } \\
\text { buildings }\end{array}$ & Moscow & Washington 1 & Washington 2 & Milan & Paris & $\begin{array}{c}\text { Moscow } \\
\text { Synthetic } \\
\text { obstacles }\end{array}$ \\
\hline Latitude & $55^{\mathrm{O}} 32^{\prime} \mathrm{N}$ & $55^{\circ} 32^{\prime} \mathrm{N}$ & $38^{0} 56^{\prime} \mathrm{N}$ & $38^{0} 54^{\prime} \mathrm{N}$ & $45^{\mathrm{O}} 27^{\prime} \mathrm{N}$ & $48^{0} 50^{\prime} \mathrm{N}$ & $55^{0} 32^{\prime} \mathrm{N}$ \\
\hline Longitude & $37^{0} 31^{\prime} \mathrm{E}$ & $37^{0} 31^{\prime} \mathrm{E}$ & $77^{0} 4^{\prime} \mathrm{W}$ & $77^{0} 2^{\prime} \mathrm{W}$ & $9^{0} 11^{\prime} \mathrm{E}$ & $2^{0} 19^{\prime} \mathrm{E}$ & $37^{0} 31^{\prime} \mathrm{E}$ \\
\hline Junctions/km2 & 59.50 & 59.50 & 66.43 & 104.64 & 189.29 & 269.75 & 59.50 \\
\hline St. length $(\mathrm{km})$ & 107.11 & 107.11 & 62.42 & 131.70 & 127.95 & 198.82 & 107.11 \\
\hline Avg. st. length (m) & 199.84 & 199.84 & 262.27 & 195.41 & 111.16 & 104.92 & 199.84 \\
\hline Obstacle coef. & 0.8398 & 0.5492 & 0.3478 & 0.5111 & 0.4809 & 0.5412 & 0.2004 \\
\hline
\end{tabular}

nal performance in a linear way. Since there are a lot of factors that can affect final performance, a generalization of these effects is difficult to achieve, but it becomes clear that the building coefficient is a factor that must be taken into account in city profiling.

Therefore, we can see that different building layouts affect the message propagation performance for very different urban environments, even if they are masked by other factors like road length. Although a complete understanding of how many different city factors actually have an impact on final performance is still lacking, it is worth getting an a priori knowledge of the performance that a city will achieve in a broadcast process. This information will allow us to select the aggressiveness, understood as number of rebroadcasting nodes, the number and periodicity of duplicated alerts, the number of RSU nodes in a DTN process, etc., required by broadcast protocols to provide effective data diffusion.

\section{Conclusions}

In this paper we rely on empirical results from real vehicular testbeds to adjust a propagation model based on the Free Space model, the Nakagami model, and an obstacle model to achieve more accurate performance results in our simulations. We evaluated the impact of this new model against other usual simulation schemes, like line-of-sight propagation models, models where no building blockage is taken into account, and models where propagation is only allowed along streets. We detected significant differences be- tween all models in terms of total vehicle reachability and delay in a broadcast process, with differences greater than $75 \%$ in the total number of reached cars.

We also checked how the obstacle model affects performance for different city profiles. We found that its influence, as a building layout component, may be presented jointly with a road layout component. We found, for our sample, that a good candidate for this combination is the street length.

As future work, we want to further explore the trend we detected to obtain a general relationship between the different city layout parameters, thus obtaining a city profiling system that is as accurate as possible, being able to get a priori knowledge of how a protocol will perform in a city. We also want to study the impact of car density on performance.

\section{Acknowledgements}

This work was partially supported by the Ministerio de Ciencia e Innovación, Spain, under Grant TIN2011-27543-C03-01, and by the Ministerio de Educación, Spain, under the FPU program, AP20104397.

\section{References}

[1] "Mobiwave - on-board equipment." [Online]. Available: http://www.savarinetworks.com/

[2] M. Boban, T. Vinhoza, M. Ferreira, J. Barros, and O. Tonguz, "Impact of vehicles as obstacles 
in vehicular ad hoc networks," Selected Areas in Communications, IEEE Journal on, vol. 29, no. 1, pp. 15-28, 2011.

[3] R. Meireles, M. Boban, P. Steenkiste, O. Tonguz, and J. Barros, "Experimental study on the impact of vehicular obstructions in vanets," in Vehicular Networking Conference (VNC), 2010 IEEE. IEEE, 2010, pp. 338-345.

[4] H. Huang, P. Luo, M. Li, D. Li, X. Li, W. Shu, and M. Wu, "Performance evaluation of suvnet with real-time traffic data," Vehicular Technology, IEEE Transactions on, vol. 56, no. 6, pp. 3381-3396, 2007.

[5] H. Stubing, M. Bechler, D. Heussner, T. May, I. Radusch, H. Rechner, and P. Vogel, "simtd: a car-to-x system architecture for field operational tests [topics in automotive networking]," Communications Magazine, IEEE, vol. 48, no. 5, pp. 148-154, 2010.

[6] M. Killat and H. Hartenstein, "An empirical model for probability of packet reception in vehicular ad hoc networks," EURASIP Journal on Wireless Communications and Networking, vol. 2009, p. 4, 2009.

[7] E. Giordano, R. Frank, G. Pau, and M. Gerla, "Corner: a step towards realistic simulations for vanet," in Proceedings of the seventh ACM international workshop on VehiculAr InterNETworking. ACM, 2010, pp. 41-50.

[8] S. Hosseini Tabatabaei, M. Fleury, N. Qadri, and M. Ghanbari, "Improving propagation modeling in urban environments for vehicular ad hoc networks," Intelligent Transportation Systems, IEEE Transactions on, vol. 12, no. 3, pp. 705$716,2011$.

[9] F. Martinez, C. Toh, J. Cano, C. Calafate, and P. Manzoni, "Realistic radio propagation models (rpms) for vanet simulations," in Wireless Communications and Networking Conference, 2009. WCNC 2009. IEEE. Ieee, 2009, pp. 1-6.
[10] C. Sommer, D. Eckhoff, R. German, and F. Dressler, "A computationally inexpensive empirical model of ieee $802.11 \mathrm{p}$ radio shadowing in urban environments," in Wireless On-Demand Network Systems and Services (WONS), 2011 Eighth International Conference on. IEEE, 2011, pp. 84-90.

[11] H. Cozzetti, C. Campolo, R. Scopigno, and A. Molinaro, "Urban vanets and hidden terminals: Evaluation through a realistic urban grid propagation model," in Vehicular Electronics and Safety (ICVES), 2012 IEEE International Conference on, 2012, pp. 93-98.

[12] R. Scopigno, H. Cozzetti, L. Pilosu, and F. Fileppo, "Advances in the analysis of urban vanets: Scalable integration of radii in a network simulator," in Wireless and Mobile Computing, Networking and Communications (WiMob), 2012 IEEE 8th International Conference on, 2012, pp. 563-570.

[13] M. Baguena, C. Calafate, J. Cano, and P. Manzoni, "Towards realistic vehicular network simulation models," in Wireless Days (WD), 2012 IFIP, 2012, pp. 1-3.

[14] D. Eckhoff, C. Sommer, and F. Dressler, "On the Necessity of Accurate IEEE 802.11p Models for IVC Protocol Simulation," in 75th IEEE Vehicular Technology Conference (VTC2012-Spring). Yokohama, Japan: IEEE, May 2012.

[15] M. Fogue, P. Garrido, F. Martinez, J. Cano, C. Calafate, and P. Manzoni, "Pawds: A roadmap profile-driven adaptive system for alert dissemination in vanets," in Network Computing and Applications (NCA), 2011 10th IEEE International Symposium on. IEEE, 2011, pp. 1-8. 